\title{
Topologically Protected Quantum Transport in Locally Exfoliated Bismuth at Room Temperature
}

\author{
C. Sabater, ${ }^{1}$ D. Gosálbez-Martínez, ${ }^{1}$ J. Fernández-Rossier, ${ }^{2}$ J. G. Rodrigo, ${ }^{3}$ C. Untiedt, ${ }^{1}$ and J. J. Palacios ${ }^{3}$ \\ ${ }^{1}$ Departamento de Física Aplicada, Universidad de Alicante, San Vicente del Raspeig, Alicante 03690, Spain \\ ${ }^{2}$ International Iberian Nanotechnology Laboratory, Avenida Mestre José Veiga, 4715-330 Braga, Portugal \\ ${ }^{3}$ Departamento de Física de la Materia Condensada and Instituto de Física de la Materia Condensada (IFIMAC), \\ Universidad Autónoma de Madrid, Cantoblanco, Madrid 28049, Spain \\ (Received 11 October 2012; revised manuscript received 12 December 2012; published 23 April 2013)
}

\begin{abstract}
We report electrical conductance measurements of $\mathrm{Bi}$ nanocontacts created by repeated tip-surface indentation using a scanning tunneling microscope at temperatures of 4 and $300 \mathrm{~K}$. As a function of the elongation of the nanocontact, we measure robust, tens of nanometers long plateaus of conductance $G_{0}=2 e^{2} / h$ at room temperature. This observation can be accounted for by the mechanical exfoliation of a $\mathrm{Bi}(111)$ bilayer, a predicted quantum spin Hall (QSH) insulator, in the retracing process following a tip-surface contact. The formation of the bilayer is further supported by the additional observation of conductance steps below $G_{0}$ before breakup at both temperatures. Our finding provides the first experimental evidence of the possibility of mechanical exfoliation of Bi bilayers, the existence of the QSH phase in a two-dimensional crystal, and, most importantly, the observation of the QSH phase at room temperature.
\end{abstract}

DOI: 10.1103/PhysRevLett.110.176802

PACS numbers: 73.22. $-\mathrm{f}, 73.63 . \mathrm{Rt}, 73.90 .+\mathrm{f}$

Topological insulators (TIs) present a gap in the bulk, but also present surface states protected against backscattering by time reversal symmetry [1]. This implies that they are immune to nonmagnetic disorder-induced localization; i.e., they are able to carry electrical current on the surface regardless of imperfections. Two-dimensional TIs [2], actually predicted before their 3D counterparts [3], are expected to exhibit the so-called quantum spin Hall (QSH) phase, a spin filtered version of the integer quantum Hall effect [4]. While the most exotic experimental manifestation of this phase is through a nearly universal spin Hall conductivity of $\approx e / 2 \pi$, a truly universal charge transport is expected to manifest, e.g., as a two-terminal conductance $G_{0}=2 e^{2} / h$.

To date, two types of 2D systems have been predicted to be QSH insulators: two-dimensional crystals such as graphene [2] or $\mathrm{Bi}(111)$ bilayers [5] and semiconductor heterojunctions such as CdTe/HgTe [6] or, more recently, InAs/GaSb quantum wells [7]. Transport measurements in $\mathrm{CdTe} / \mathrm{HgTe}$ [6] and InAs/GaSb [8] quantum wells have revealed the presence of protected edge states and provided the first experimental evidence of the QSH phase to date. The QSH state in 2D crystals, on the other hand, has not been experimentally confirmed to date. The fact that spinorbit coupling (SOC) in graphene is so weak precludes the observation of the QSH phase in this material. Bismuth, on the contrary, presents a naturally large SOC, its mechanical and electronic properties are well characterized for bulk and surface [9], in nanowire form [10], and, recently, the existence of edge states in $\mathrm{Bi}(111)$ bilayers has been reported [11]. Other proposals stay, at this moment, at a more speculative level [12].
Cleavage techniques are becoming common in the quest for 2D crystals [13]. With a few exceptions [14], they remain largely unexplored in the field of TIs. We report here, using scanning tunneling microscope (STM) based mechanical and electrical characterization techniques, the first evidence of the QSH phase in a two-dimensional crystal such as an exfoliated $\mathrm{Bi}(111)$ bilayer. The procedure follows closely that of creation of atomic size metallic contacts [15], namely, repeated indentations of a tip against a surface while measuring the current at low voltages (typically $100 \mathrm{meV}$ ). The resulting conductance traces contain information on the atomic and electronic structure of the contact. The experiments where done at room temperature (and ambient conditions) and at low temperatures (4 K) in cryogenic vacuum using two Bi samples of different origin [16]. Related experiments have been reported in the past [17-19], lacking a convincing interpretation of the results, but have, in part, motivated our work.

As a rule of thumb (in particular, for monovalent elements) each atom forming the minimum cross section of a metallic nanocontact contributes to the conductance with a value in the vicinity of $G_{0}$ [15]. Thus, on retracing the tip after indentation, one-atom cross-section contacts formed right before complete breakup are signaled by a conductance plateau (as a function of the STM piezotube elongation) near the quantum of conductance $G_{0}$. Unlike their more common metallic counterparts, the conductance traces of $\mathrm{Bi}$ nanocontacts at $4 \mathrm{~K}$ present several subplateaus below $G_{0}$ before breakup [see Fig. 1(a)]. In Fig. 1(b), we have singled out one trace that manifestly shows four subplateaus before the final rupture. Since each plateau corresponds to an elastic deformation of an atomic 

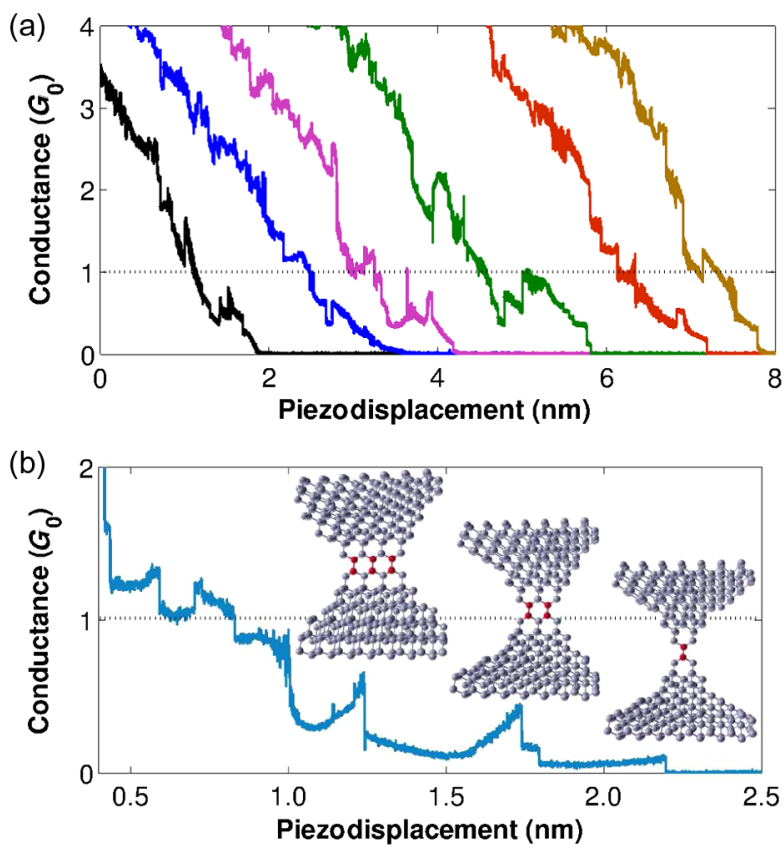

FIG. 1 (color online). (a) Selected curves obtained during the process of rupture of a $\mathrm{Bi}$ nanocontact formed after indentation of a tip on a surface at $4 \mathrm{~K}$. (b) Detail of a selected curve where four conductance subplateaus can be appreciated below $G_{0}$. The insets represent possible atomic configurations compatible with the subplateau values.

configuration in between plastic deformation events, one can only conclude that few-atom cross-section Bi nanocontacts (as the ones shown in the insets) are much less conducting than their metallic counterparts. As it is a common practice to record conductance histograms to statistically characterize nanocontacts [15], we have done so using thousands of rupture traces. We find a large statistical weight below $G_{0}$, but no characteristic values can be appreciated [16].

In air at $300 \mathrm{~K}$ the mechanical stability of the experimental setup is inferior to that at low temperatures, making it more difficult to carry out measurements in a systematic way. Previously, the samples were fully characterized [16], paying attention to surface contamination and, in particular, to oxidation. This turned out to be very small, as has been long known for Bi [20]. Starting from conductance values at a maximum indentation of the order of $10 G_{0}$, most of the traces either do not break for the allowed range of the piezotube, typically up to $300 \mathrm{~nm}$, or fall to zero showing no apparent reproducible behavior. Starting from smaller indentations (up to 3-6G 0 ), however, consecutive traces may repeatedly show small features or short plateaus, including plateaus at $G_{0}$ [see Fig. 2(a)] which were completely absent in the $4 \mathrm{~K}$ measurements. Notice also the different length scales in Figs. 1(a) and 1(b) (1 nm) and Figs. 2(a) and 2(b) (100 nm). Most notably, long conductance plateaus appear on occasion on some traces [see Fig. 2(b)], the length of these reaching up to hundreds
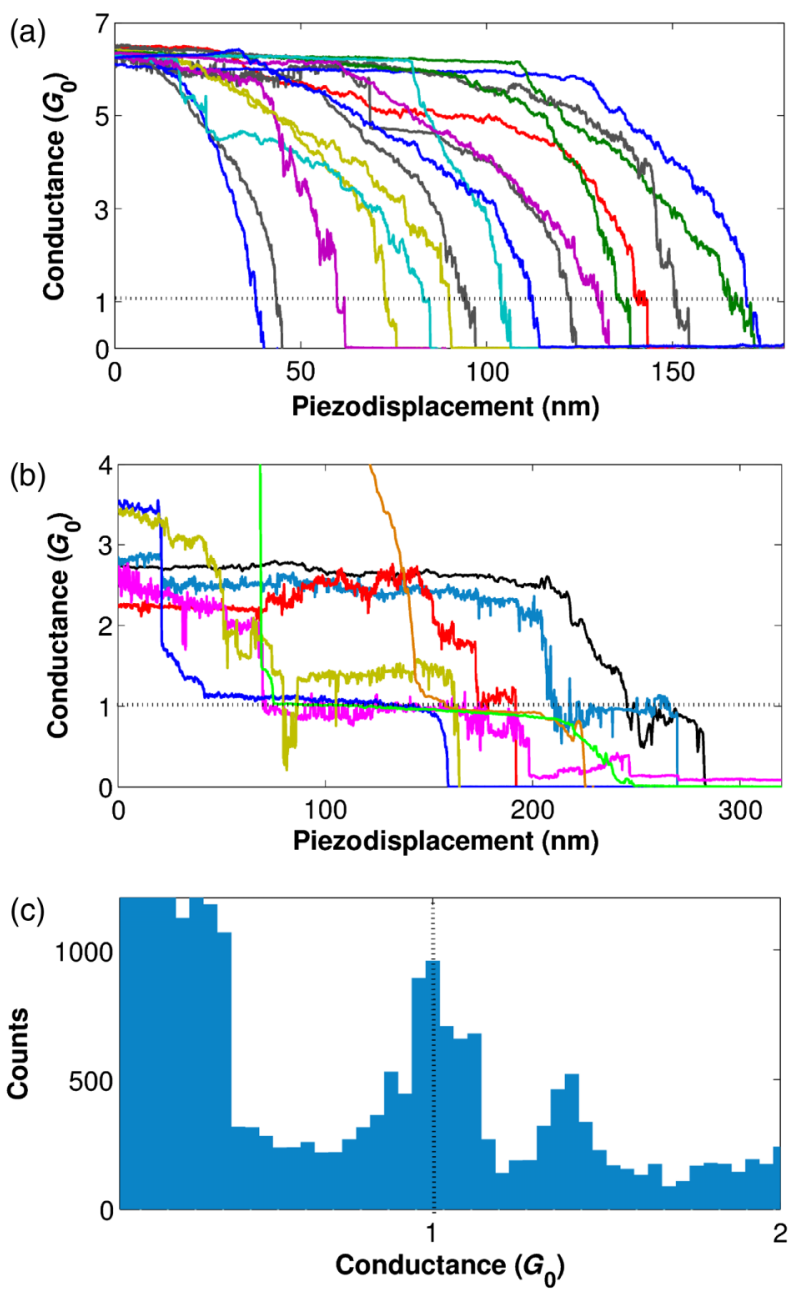

FIG. 2 (color online). (a) Examples of conductance recorded during the process of rupture of a $\mathrm{Bi}$ nanocontact formed by repeated indentation of a $\mathrm{Bi}$ tip on a $\mathrm{Bi}$ surface at $300 \mathrm{~K}$. (b) Conductance traces exhibiting plateaus with elongations of tens and hundreds of nanometers attributed to the occasional exfoliation of large flakes of $\mathrm{Bi}(111)$ bilayers between tip and substrate. Most of the plateaus are pinned at $G_{0}$. (c) Histogram of conductance obtained from tens of traces exhibiting long plateaus. The histogram exhibits a clear peak at $G_{0}$, which reveals that this value is particularly frequent and robust.

of nanometers. While some of them correspond to the initial elongation elastic process (the ones around $3 G_{0}$ ), most of them appear after a few plastic events. One way to quantify the information in these traces is to plot a conductance histogram only with them (we have selected those exhibiting plateaus longer than $50 \mathrm{~nm}$ regardless of the plateaus' conductance values). The result is incontrovertible, showing a large peak at $G_{0}$ since most of the plateaus are largely pinned at the quantum of conductance [see Fig. 2(c)].

An exact conductance value of $G_{0}$ is generally expected to occur when, first, the Fermi wavelength is comparable to the constriction width and, second, the constriction potential is adiabatic in the current direction. This happens, for 
instance, in a gated two-dimensional electron gas [21] or in monovalent metal atomic contacts [15]. Crystalline bulk Bi presents two types of carriers (electrons and holes) with long Fermi wavelengths (tens of $\mathrm{nm}$ ) and unusually small masses [22]. A first scenario, already put forward to explain the early experiments in Ref. [18], invokes the formation of large cross section three-dimensional constrictions (thousands of atoms) so that the lateral movement of the longwavelength electrons gets quantized. It is, however, difficult to predict how the bulk electronic structure can carry over to constrictions presumably lacking long crystalline order. In addition, adiabaticity imposes severe restrictions to the shape of the constrictions. Furthermore, it is quite hard to imagine how these constrictions may be stable during very long piezodisplacements. Even more difficult to accept is the fact that they must exist right before rupture without then going through a very large number of plastic deformation events. A second scenario where atom-size constrictions are responsible for the appearance of $G_{0}$ is ruled out by the observation of subquantum plateaus in most traces (see Figs. 1 and 2) and also by our calculations (see below).

We now give our rationale to our findings. Bulk Bi can be viewed as a layered material in the (111) direction [16], much as graphite, but with a stronger electronic coupling between layers. In fact, a $\mathrm{Bi}(111)$ bilayer is similar to graphene in many respects, in particular, the atomic structure is that of a puckered honeycomb lattice, with the atoms of one sublattice shifted perpendicularly with respect to those of the other sublattice. A Bi(111) bilayer has been predicted to be a QSH insulator [5] with a large bulk gap and three pairs of helical edge states [23]. This odd number of edge states guarantees that the transport properties of a $\operatorname{Bi}(111)$ flake should be robust against size, shape, and weak disorder. In particular, it should generically exhibit a universal two-terminal conductance of $G_{0}$.

Given the layered structure of $\mathrm{Bi}$, the general picture that naturally emerges from our experiments is that of a contact-induced exfoliation of a $\mathrm{Bi}(111)$ bilayer [see Figs. 3(a) and 3(b)]. We first note that the interbilayer coupling is about 10 times weaker than the intrabilayer covalent bonding [9]. The $\operatorname{Bi}(111)$ surface presents a Debye temperature in the $70-80 \mathrm{~K}$ range [24], so that at ambient conditions it should not come as a surprise that, once a gentle contact is made on the appropriate surface orientation, retracing the tip can peel off a single bilayer. One extreme of this bilayer (while the rest remains in contact with the surface) sticks to the tip which now acts as an electrode [see Figs. 3(a) and 3(b) and the movie in the Supplemental Material [16]]. The specifics of the proposed mechanical exfoliation are unknown to us, being unclear whether the tip breaks the surface or simply elastically deforms it after indentation, and whether slip directions on the (111) planes favor slipping over detaching mechanisms. The number of atoms participating in the contact at maximum conductance is also unknown, but it remains (a)

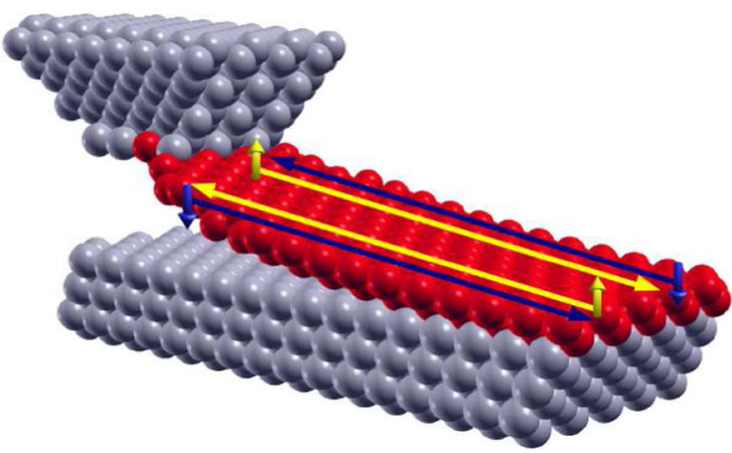

(b)
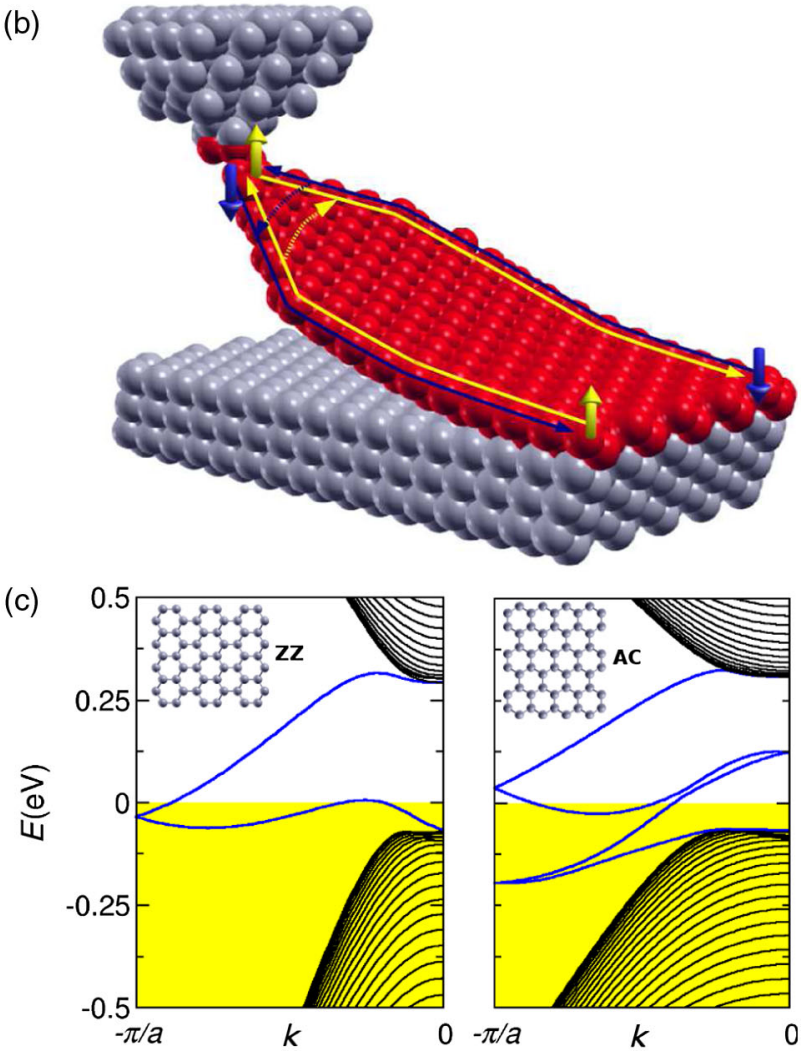

FIG. 3 (color online). (a) Pictorial representation of the proposed process of exfoliation of a $\mathrm{Bi}(111)$ bilayer [dark gray (red)] after contact with the STM tip. One (out of three) helical edge channel is also represented. (b) Same as in (a) but for the last stages of piezotube elongation before breakup where a fewatom constriction forms. (c) Band structure of $\mathrm{Bi}(111)$ bilayer nanoribbons along $\mathrm{ZZ}$ (left) and $\mathrm{AC}$ (right) directions as obtained from a self-consistent tight-binding model. The insets show a top-view section of these nanoribbons. The edge bands connecting valence and conduction bands are shown by blue solid lines. The Fermi energy (set to zero) crosses three times these edge bands which gives rise to three conduction channels.

large enough after exfoliation so that the theoretically expected intrinsic conductance $G_{0}$ of the bilayer is not masked by the contact resistance.

With this picture in mind, the conductance subplateaus below $G_{0}$ can only reflect the breaking of the tip-bilayer contact or the progressive breaking of the bilayer, a process in which a bidimensional nanocontact may form 

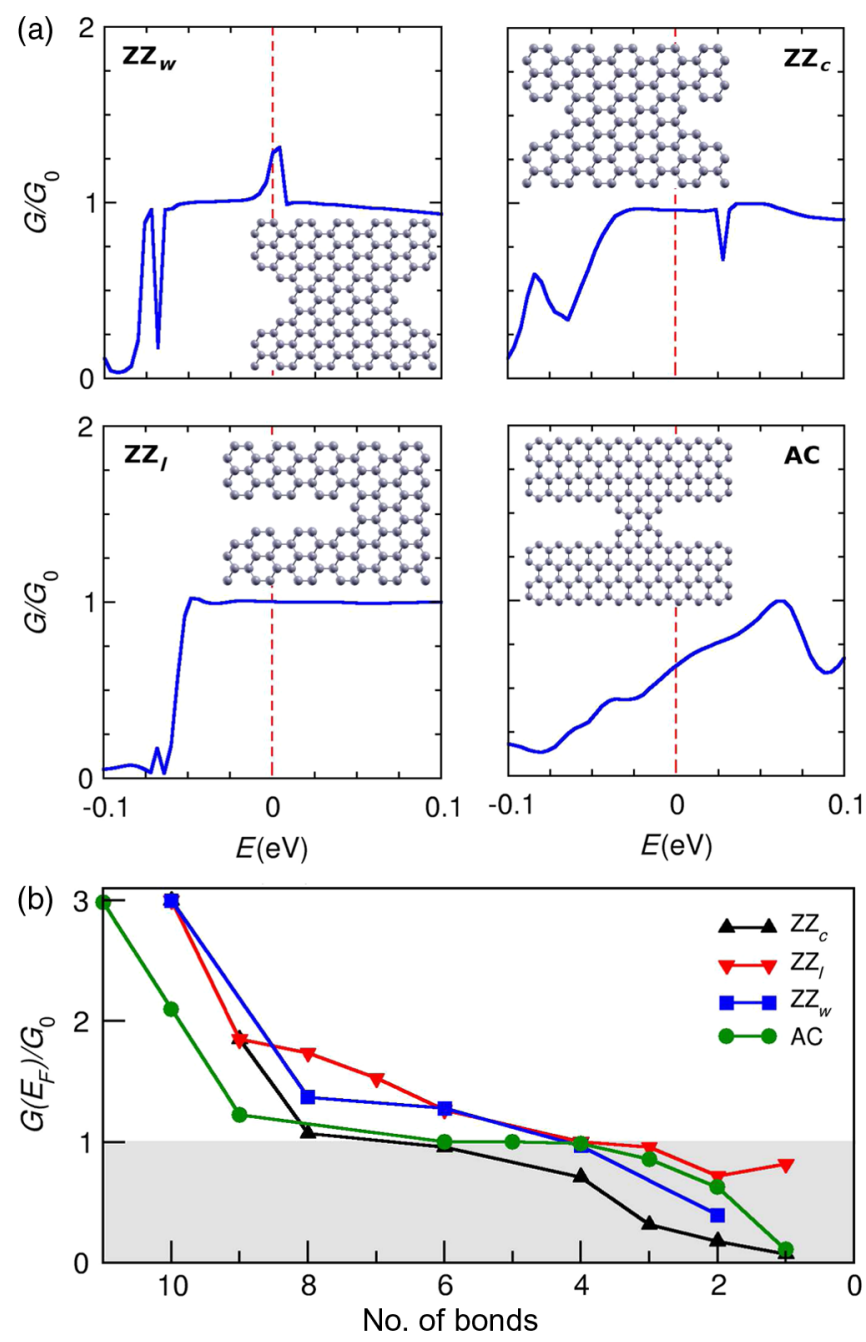

FIG. 4 (color online). (a) Conductance versus electron energy for four constriction models in $\mathrm{ZZ}$ and $\mathrm{AC} \mathrm{Bi}(111)$ bilayer nanoribbons. (b) Conductance at the Fermi energy for many cases as those in (a) as a function of the number of bonds remaining in the constriction. The subindexes denote central(c), lateral-(l), and wedge-type (w) constrictions.

[see Fig. 3(b) and insets in Fig. 4(a)]. Since a disordered $\mathrm{Bi}(111)$ bilayer is expected to exhibit a maximum conductance of $G_{0}$, we can only expect the nanocontacts thus formed to conduct less due to interedge backscattering at the constriction (this is more clearly observed at $4 \mathrm{~K}$ ). Noticeably, at $4 \mathrm{~K}$, long plateaus do not appear near $G_{0}$. We attribute this absence to the stronger interbilayer binding at low temperatures which prevents the exfoliation of a sufficiently large flake. Interestingly, the upturns of the subplateaus rarely surpass $G_{0}$.

We now support our hypothesis with atomistic calculations. We model $\mathrm{Bi}$ with a four-orbital tight-binding Hamiltonian with the parametrization given by Liu and Allen [22]. In order to account properly for the electronic structure at the edges, we carry out self-consistent calculations including local electron-electron interactions [16]. As can be seen in Fig. 3(c), Bi(111) bilayer nanoribbons grown along zigzag (ZZ) and armchair (AC) crystallographic orientations present a gap with valence and conduction bands connected in a continuous manner by an edge state band. This edge band cuts three times the Fermi energy and presents the same topology as that computed with more sophisticated density functional theory calculations [23], which gives us confidence in our model. We have also verified that the nature of the wave functions in this band follows that expected in a QSH insulator [16].

Equipped with a reliable atomistic description of $\mathrm{Bi}$, we now perform coherent quantum transport calculations. (While inelastic effects cannot be entirely ruled out, bulk Bi carriers are known to have very long mean free paths even at room temperature [25].) We introduce constrictions in the nanoribbons emulating the breaking of the bilayer flake [see insets in Fig. 4(a). For the sake of simplicity, we have chosen to perform this type of calculation instead of the one for a contact between a tip (of unknown shape) and the flake [as shown in Figs. 3(a) and 3(b)]. A common way to calculate the conductance (as a function of energy) $G(E)$ is through the Green function formalism and the partitioning technique [16,26,27], now taking fully into account the atomic SOC [16,28]. Results of $G(E)$ for four different constriction geometries in $\mathrm{AC}$ and $\mathrm{ZZ}$ nanoribbons are shown in Fig. 4(a). Figure 4(c) shows $G$ at the Fermi energy $\left(E_{F}=0\right)$ for these four geometries as a function of the number of bonds at the constriction (i.e., as a function of the width). Only in disorder-free ribbons with perfectly defined edges the three channels can fully transmit. For constriction widths just two or three bonds smaller than that of the ribbons, the conductance drops in all cases to values in the close vicinity of $G_{0}$. Only constrictions featuring less than approximately five bonds conduct below $G_{0}$. (The robust appearance of $G_{0}$ also holds for other types of disorder [16].) These results can be simply understood in terms of strong intraedge backscattering of two of the edge channels and absent or partial interedge backscattering of the remaining helical pair at the narrowest section of the constriction [see Figs. 3(a) and 3(b)]. Our estimate of a minimum of five bonds for a constriction to support $G\left(E_{F}\right)=G_{0}$ is consistent with the maximum number of plastic events typically seen in the experiments below $G_{0}$ before the constriction definitely breaks [see, e.g., Fig. 1(b)].

In summary, we have reported and offered a consistent interpretation of the appearance of extremely long quantum of conductance plateaus in the breaking process of $\mathrm{Bi}$ nanocontacts. We attribute it to the occasional local exfoliation of Bi bilayers, predicted to be 2D TIs. Other interpretations cannot be ruled out, but should account for three extraordinary facts: (i) the quantum of conductance cannot be associated with a single-atom contact, but with a nanoscopic constriction, (ii) it appears at room temperature, and (iii) it is robust to small concentration of contaminants since the experiments are performed in air. 
The first two authors, C.S. and D. G.-M., contributed equally to this work, the former on the experimental side and the latter on the theoretical part. We thank the Spanish MICINN for financial support through Grants No. FIS201021883, No. FIS2011-23488, and No. CONSOLIDER CSD2007-0010, and Generalitat Valenciana through Grants No. ACCOMP/2012/127 and No. PROMETEO/ 2012/011. D. G.-M. acknowledges the Centro de Computación Científica at UAM for computational support. Cristina Almansa is gratefully acknowledged for her help with the transmission electron microscope.

[1] M.Z. Hasan and C. L. Kane, Rev. Mod. Phys. 82, 3045 (2010).

[2] C. L. Kane and E. J. Mele, Phys. Rev. Lett. 95, 226801 (2005).

[3] H. Zhang, C.-X. Liu, X.-L. Qi, X. Dai, Z. Fang, and S.-C. Zhang, Nat. Phys. 5, 438 (2009).

[4] The Quantum Hall Effect, edited by R. E. Prange, S. M. Girvin, and K. v. Klitzing (Springer, New York, 1986).

[5] S. Murakami, Phys. Rev. Lett. 97, 236805 (2006).

[6] M. König, S. Wiedmann, C. Brüne, A. Roth, H. Buhmann, L. W. Molenkamp, X.-L. Qi, and S.-C. Zhang, Science 318, 766 (2007).

[7] C. Liu, T. L. Hughes, X.-L. Qi, K. Wang, and S.-C. Zhang, Phys. Rev. Lett. 100, 236601 (2008).

[8] I. Knez, R.-R. Du, and G. Sullivan, Phys. Rev. Lett. 107, 136603 (2011).

[9] P. Hofmann, Prog. Surf. Sci. 81, 191 (2006).

[10] Z. Zhang, D. Gekhtman, M. S. Dresselhaus, and J. Y. Ying, Chem. Mater. 11, 1659 (1999).

[11] F. Yang, L. Miao, Z. F. Wang, M.-Y. Yao, F. Zhu, Y. R. Song, M.-X. Wang, J.-P. Xu, A. V. Fedorov, Z. Sun, G. B. Zhang, C. Liu, F. Liu, D. Qian, C. L. Gao, and J.-F. Jia, Phys. Rev. Lett. 109, 016801 (2012).

[12] C.-C. Liu, W. Feng, and Y. Yao, Phys. Rev. Lett. 107, 076802 (2011).
[13] B. Radisavljevic, A. Radenovic, J. Brivio, V. Giacometti, and A. Kis, Nat. Nanotechnol. 6, 147 (2011).

[14] S. S. Hong, W. Kundhikanjana, J. J. Cha, K. Lai, D. Kong, S. Meister, M. A. Kelly, Z.-X. Shen, and Y. Cui, Nano Lett. 10, 3118 (2010).

[15] N. Agraït, A. L. Yegati, and J. M. van Ruitenbeek, Phys. Rep. 377, 81 (2003).

[16] See Supplemental Material at http://link.aps.org/ supplemental/10.1103/PhysRevLett.110.176802 for details.

[17] J. M. Krans and J. M. van Ruitenbeek, Phys. Rev. B 50, 17659 (1994).

[18] J. G. Rodrigo, A. García-Martín, J. J. Sáenz, and S. Vieira, Phys. Rev. Lett. 88, 246801 (2002); S. Sangiao, J. M. Michalik, L. Casado, M. C. Martínez-Velarte, L. Morellón, M. R. Ibarra, and J.M. De Teresa, Phys. Chem. Chem. Phys. 15, 5132 (2013).

[19] J. L. Costa-Krämer, N. García, and H. Olin, Phys. Rev. Lett. 78, 4990 (1997).

[20] R. M. Tahboub, M.E. Guindy, and H. D. Merchant, Oxid. Met. 13, 545 (1979).

[21] B. J. van Wees, L.P. Kouwenhoven, E. M. M. Willems, C. J. P. M. Harmans, J. E. Mooij, H. van Houten, C. W. J. Beenakker, J. G. Williamson, and C. T. Foxon, Phys. Rev. B 43, 12431 (1991).

[22] Y. Liu and R. E. Allen, Phys. Rev. B 52, 1566 (1995).

[23] M. Wada, S. Murakami, F. Freimuth, and G. Bihlmayer, Phys. Rev. B 83, 121310 (2011).

[24] M. Mayrhofer-Reinhartshuber, A. Tamtögl, P. Kraus, K. H. Rieder, and W. E. Ernst, J. Phys. Condens. Matter 24, 104008 (2012).

[25] V.D. Das and N. Soundararajan, Phys. Rev. B 35, 5990 (1987).

[26] J. J. Palacios, D. Jacob, M. Soriano, A. J. Pérez-Jiménez, E.S. Fabián, E. Louis, and J.A. Vergés, ALACANT quantum transport package, http://alacant.dfa.ua.es.

[27] D. Jacob and J. J. Palacios, J. Chem. Phys. 134, 044118 (2011).

[28] D. Gosálbez-Martínez, J. J. Palacios, and J. FernándezRossier, Phys. Rev. B 83, 115436 (2011). 